\title{
Research on Penetration Behavior and after effects of Coated Reactive Fragments Impacting Steel targets
}

\author{
J Peng*, B H Yuan, X Y Sun, Y J Chen, H Chen \\ Xi'an Modern Chemistry Research Institute, Xi'an, China
}

\begin{abstract}
For research the behavior and after effects which had been resulted from the coated reactive fragments impacting the steel targets, the $14.5 \mathrm{~mm}$ ballistic rifle had been adopted to complete the experiments that the $6 \mathrm{~mm}$ Q235A steel targets was impacted by the steel fragments and the reactive fragments, composed by metal/ polymer coated with steel, with the same mass and size. Afterwards, the v50 ballistic-limited velocity and the law of the damage effects on the after effect-targets had been discovered. The results indicate that the integral strength of the coated reactive fragments was lower than that of the inert steel fragments, which lead to the reductions of the penetration performance. The $\mathrm{v} 50$ ballistic-limited velocity, resulted of the coated reactive fragments impacting the $6 \mathrm{~mm}$ Q235A steel targets, was $709 \mathrm{~m} / \mathrm{s}$ and it was 1.33 times than that of the steel fragments with the same mass and size. The reaming enhanced effects on the after effecttargets could be produced in the condition that the impact velocity of coated reactive fragments should be greater than $900 \mathrm{~m} / \mathrm{s}$ while the residual velocity should be greater than $181 \mathrm{~m} / \mathrm{s}$, and that it had been strengthened along with the impact velocity increased. The probable reasons of the damage enhanced effects had been analyzed that the coupled damage effects had been resulted from two parts energy. The results will provide important references to the engineering application of reactive fragments for warheads.
\end{abstract}

\section{INTRODUCTION}

Reactive fragments are one type of energetic destroying elements that energy release characteristics can be triggered by the impact. Other than the traditional inert fragments, the speedy combustion or explosion effects will be produced while the reactive fragments impacting the targets, with the result that the damage power of weapon warhead can be improved significantly. Accordingly, the reactive fragments technology and the invadeexplode characteristics are identified as the advanced technology and research hotspot in the field of the high efficiency damage ammunition [1-3].

The formulation and preparation technics of the reactive materials, mechanical properties, reaction energy-released characteristics, reaction mechanisms and damage effects are several important aspects of reactive fragments technology[4]and the study on the damage effects of the reactive fragments on the targets is related to its engineering application, and that the design of reactive fragments can be guided helpfully to the research on the damage effects.

*Corresponding Author: 285649306@qq.com 
The damage effects, which was resulted in two kinds of metal/polymer reactive materials based on different metals ( $\mathrm{Al}$ and $\mathrm{Ti}$ ) impacting the steel targets, had been carried out by JunFeng Shuai [5]to obtain the impact energy-released effect of the reactive fragments and the reaming effects on the steel targets. Zhong-hua [6] Lu used explosive gun device to study the penetration characteristics of metal polymer fragments impacting medium-thick aluminum targets. The ballistic rifle experimental device had been used by Jin Chen [7] to study the penetration performance of reactive fragments impacting steel targets, and the empirical relationship of the ballistic limit velocity, resulted from reactive fragments impacting steel targets, had been obtained and that the differences between such reactive fragments and steel fragments had been analyzed. The ballistic rifle device had been used also by F.Y.Xu [8]to study the penetration effects of the metal/polymer cylindrical reactive fragments which had been formed by cold pressing and sintering on aluminum targets of different thickness, as a result the semi-empirical analysis model of ballistic limit for reactive fragments had been obtained and the mechanisms of influence on penetration capability from the reaction behavior of reactive fragments had been analyzed. The corresponding study on the damage effects of the reactive fragments impacting targets had been carried out by Brett Sorensen [9] et al. The limitations of these studies were known as that the research results and the actual experimental results of warheads explosion was mismatching. These defects were caused by that the "exposed" reactive fragments by the reactive materials directly or coated simply had been tested, therefore the mechanical and structure strength of the reactive fragments was weakened. It was applied to antipersonnel warheads and they had been broken, disintegrated and reacted before reaching targets thus the damage abilities had been lost.

Based on the above factors, the reactive fragments composed by the reactive materials of metal/ polymer coated with steel had been designed in this study and then they had better mechanical strength and chemical stability than above-mentioned "exposed" reactive fragments by the reactive materials directly or coated simply. Besides, the results of the warheads static explosive experiments showed that the initial velocity obtained of the reactive fragments could up to $2000 \mathrm{~m} / \mathrm{s}$ and remained intact after been impacted by warheads explosion, what's more the energy-released of reaction, triggered by impacting targets, could meet the tactical requirements. As a result, the reactive fragments have a good application prospect in fragment warheads. The ballistic rifle systems had been adopted to study the penetration characteristics of the coated reactive fragments with steel and the inert steel fragments penetrating steel targets. The ballistic limit velocity and aftereffect damage on the targets had been analyzed contrastively crossing the above two dimensions. Further, the mechanisms of the damage enhanced effects had been expounded based on the experimental phenomena and results analysis, in order to provide certain references for the design of the reactive fragment warheads.

\section{EXPERIMENT DESIGN}

\subsection{Experimental samples}

The structure, formed inner reactive materials coated the outer steel shell had been adopted for the coated reactive fragments, which had been prepared by the process of preparation of the reactive materials, molding[4] and encapsulation, etc. The experimental sample was shown in Figure 1. The mass of coated reactive fragments was about $10 \mathrm{~g}$, the outer diameter was $12 \mathrm{~mm}$ and the height was $12 \mathrm{~mm}$. a metal/polymer system, composed of 
$\mathrm{Al} / \mathrm{PTFE} / \mathrm{W} /$ other reactive metals, had been adopts for the reactive materials. The outer diameter was $9 \mathrm{~mm}$, the height was $9 \mathrm{~mm}$, the mass was $4.4 \mathrm{~g}$ and the density was the same as steel. The low carbon steel fragments was counted as the inert fragments, with the same mass and size as the coated reactive fragments.

The experimental targets plates had been made of Q235A steel, the size of them was $200 \mathrm{~mm} \times 200 \mathrm{~mm}$, the thickness was $6 \mathrm{~mm}$. The after-effect aluminum targets with the same size and $3 \mathrm{~mm}$ thickness, made of 2A12 aluminum alloy, had been placed at the back of the steel targets $200 \mathrm{~mm}$ away.

\subsection{Experimental devices}

The experimental devices had been composed of $14.5 \mathrm{~mm}$ ballistic rifle, cartridge holder, fragments, velocity measurement targets, double-layer spacing targets, retroreflector and high-speed camera. The schematic diagram of the experimental devices was shown in Figure 2. The launching velocity of the reactive fragments could be controlled with the propelling charge of the ballistic rifle and the velocity of fragments impacting targets could be calculate with the velocity measurement targets system.

\subsection{Experimental conditions}

The velocity range of the experimental fragments impacting the targets was $500 \sim 1400 \mathrm{~m} / \mathrm{s}$, what was the range of launching velocity for the ballistic rifle.

Multiple damage effects, composed shock wave and thermal damage and others, which had been produced by the chemical reactions, caused by the reactive fragments impacting the targets. Therefore, in this study, the perforation diameter or area of the targets and lifted altitudes in targets side, measured by Vernier calipers or image processing software, had been regarded as the parameters to indicate damage-enhanced effects and the measurement methods was shown in Figure 3. At the same time, the firing phenomenon of bullet-target impacting had been observed by high-speed camera and the duration of the flame had been recorded, which had been taken as the parameters of the chemical reactions and the severity of the reactions of the reactive fragments.

A total of 21 times experiments had been completed effectively, including the inert steel 7 times and reactive fragments 14 times

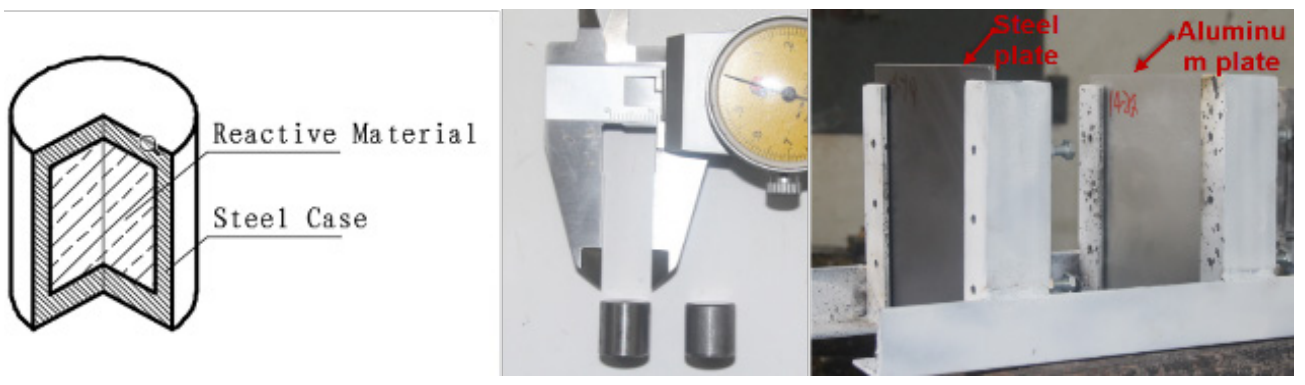

Figure 1: The reactive fragments and double-layer spacing targets 


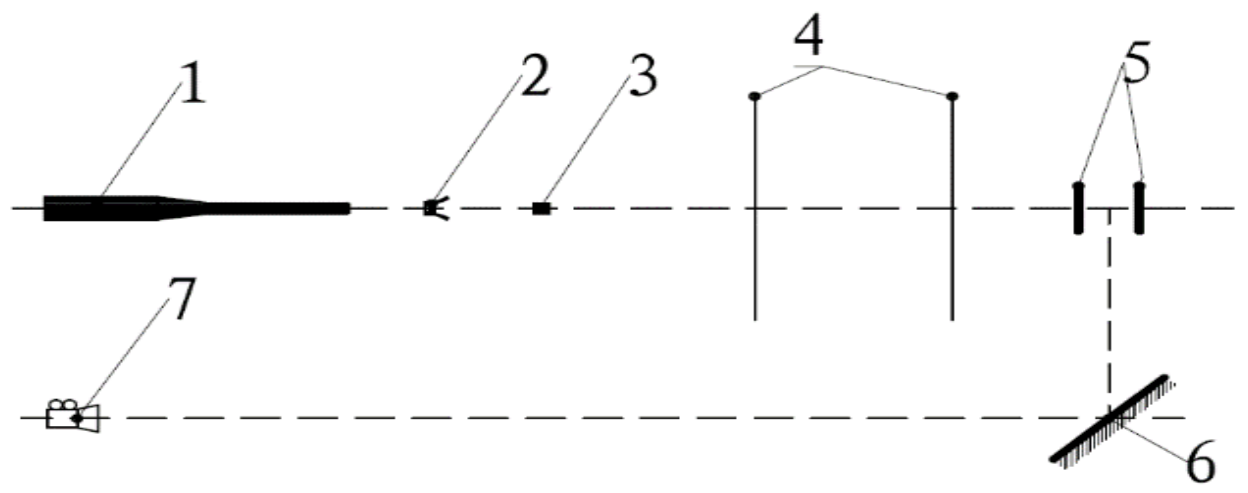

1-ballistic rifle, 2- cartridge holder, 3- fragments, 4- velocity measurement targets, 5-targets, 6- retroreflector, 7- high-speed camera

Figure 2: Schematic diagram of experimental devices
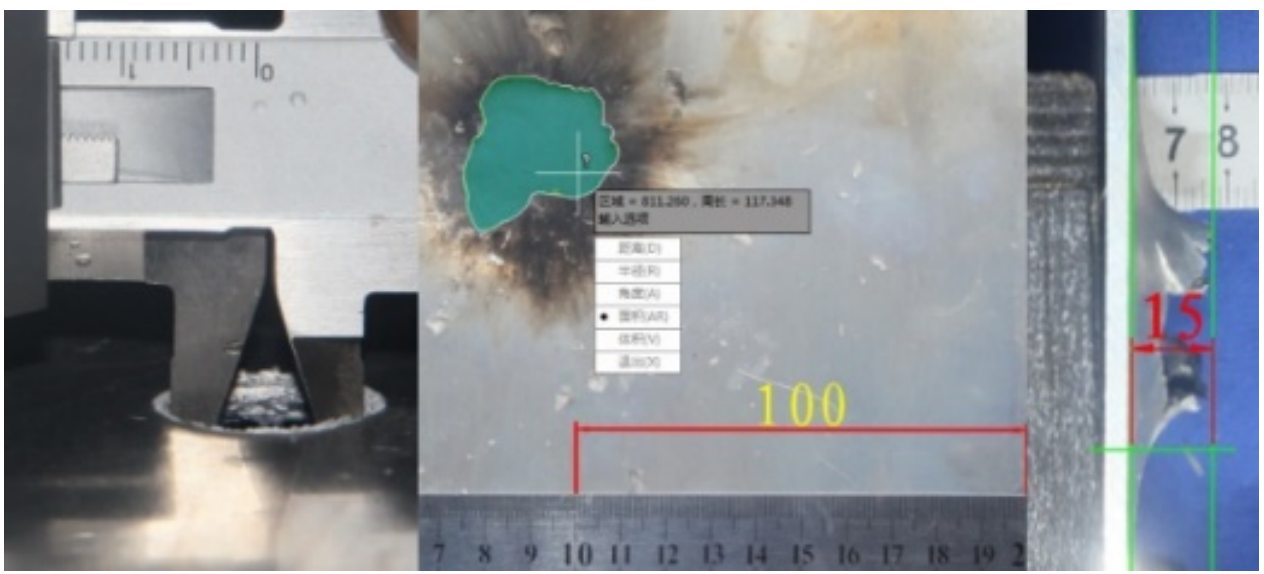

Figure 3: The Measurement method of experiment results

\section{RESULT AND DISCUSSION}

3.1 Analysis for the processes of the fragments penetrating the targets In this study, the processes and phenomena of the reactive fragments penetrating the targeted plates had been recorded by the high-speed camera. In order to obtain the sharper images, the frame frequency of shooting had been improved as much as possible under the conditions of field light(the frame frequency of high-speed camera had been set at 3000 frames/s and the exposure time at 330us). The images of fragments penetrating the targets under three typical working conditions at different times was shown in Figure 4.

The circumstances, the targets could not be penetrated by fragments was shown in Figure 4 (a). In this instance, the velocity of the fragments impacting the targets(v) was $593 \mathrm{~m} / \mathrm{s}$ and 
bright flame was generated in front of the steel targets for a about $6.6 \mathrm{~ms}$ duration. Obviously, the targeted plates could not be penetrated by fragments, but certain reactions had been initiated by the impact. The duration of the flames which had been generated by the process of the inert steel fragments penetrating the targets was extremely short, almost synchronized with the generation process, and the time scales was about tens microseconds. The flames, generated by the process of the inert steel fragments penetrating the targets, could be can observed hardly under the frame frequency of high-speed camera in this experiment. Therefore, it also was ignited and reacted although the targets could not be penetrated what would be verified furtherly from the image analysis of the damage effects on the targeted plates.

The circumstances, the targets had been penetrated by fragments was shown in Figure 4 (b). In this instance, the velocity of the fragments impacting the targets(v) was $731 \mathrm{~m} / \mathrm{s}$ and bright flame was generated between the steel and aluminum targets for a about $16.5 \mathrm{~ms}$ duration while that was not appeared in the after-effect targets. The velocity of the fragments impacting the targets was low and it should be near the fragments ballistic-limited velocity.

The circumstances, the targeted plates had been penetrated completely by fragments was shown in Figure 4 (c). In this instance, the velocity of the fragments impacting the targets(v) was $1249 \mathrm{~m} / \mathrm{s}$ and bright flame was generated between the steel and aluminum targets for a about $99.9 \mathrm{~ms}$ duration. The duration and brightness of the flames was closely related to the impact velocity and that could been improved markedly with the impact velocity increase. The duration of the flames could reach to $133 \mathrm{~ms}$ when the impact velocity of fragments was $1410 \mathrm{~m} / \mathrm{s}$

It was considered that the completeness of the reactions was determined by velocity of the fragments impacting the targets. The degree of erosion and damage for the fragments was positive correlated with the impact velocity. The results of the study[8] showed that when the degree of erosion and damage of the shell reached a certain level what was insufficient to the constraint the internal reactive materials effectively, the completeness of the reactions was improved along with the impact velocity increase, thereby forming a longer duration of the flames. It will be predicted evidently that the completeness of the reactions can be maximized when the impact velocity reaches a certain upper limit threshold velocity what will require the further researches to ascertain, due to the restrictions from the upper limit of the launching velocity of the experimental devices in this study.

\subsection{Damage effects on the targets}

\subsubsection{Damage effects on the steel targets}

The typical images of the holes, penetrated with the two types of fragments, was shown in Figure 5. The results of reactive fragments penetration was correspond to the images which had been recorded by the high-speed camera in Figure 4. As shown in the figure, a large radial deformation had been resulted in the processes of the reactive fragments penetrating the steel targets and the head section structure of the coated shell had been damaged, moreover the body section had been turned over backwards. It was similar to inert steel fragments in that the axial upsetting had been resulted in the processes of the reactive fragments penetrating the targets and shear damage had been resulted from the reverse motion of the outside edge of the upsetting. At this point, the strength of the contact center with the targets played the great significant roles in the penetration ability. However, the penetration ability of the reactive 
fragments had been weakened than that of the inert steel fragments since the strength of the reactive materials in the center of the reactive fragments was much lower than that of steel. In the circumstances that the targets could not be penetrated by the fragments, the black traces had been presented on the contact edge between the fragments and the targets, indicating that ignition reaction had been triggered in the reactive materials at the head of the reactive fragments during the penetration processes, and what could also been verified from the flames shown in Figure 4 (a).

The diameter of penetrated holes of the targets is an important indicator, which is generally used to characterize the damage power of fragments. The diameter data of penetrated holes by these experiments was shown in Table 1 .

In Table I, $\mathrm{v}$ represented the velocity of the fragments impacting the targets, $\mathrm{d}$ represented the diameter of penetrated holes to the targets, and $\mathrm{d} 0$ represented the initial diameter of the fragment. In order to characterize the increment of the diameter of penetrated holes relative to the initial diameter of the fragments, command that.

$$
N=d / d_{0}
$$

$\mathrm{N}$ represented the multiples of the aperture, resulted from the fragments impact the targets, to the initial diameter of the fragments. It could be known that the aperture was $1.35 \sim 1.65$ times to the initial diameter of the reactive fragments, and that was 1.21 1.4 times to the steel fragments.

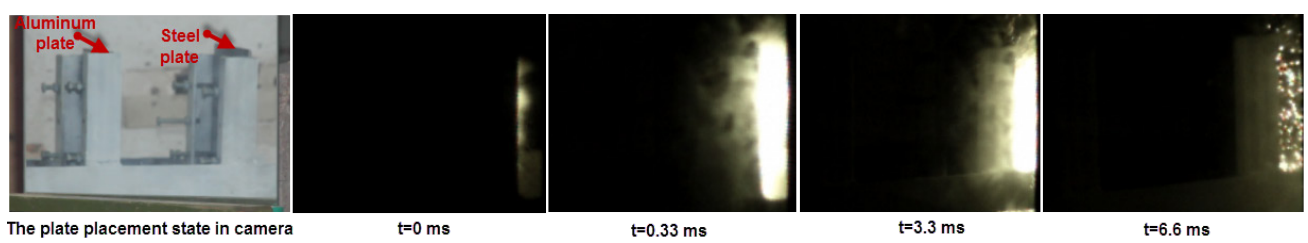

(a) $v=593 \mathrm{~m} / \mathrm{s}$

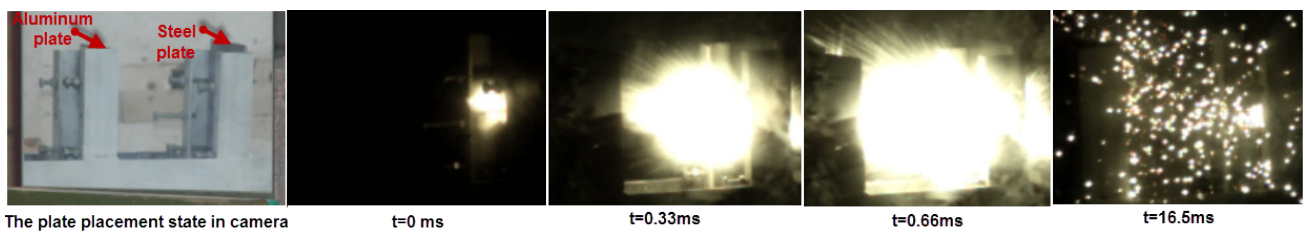

(b) $v=731 \mathrm{~m} / \mathrm{s}$

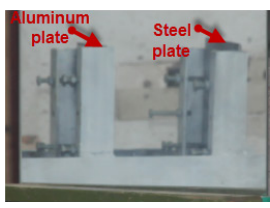

The plate placement state in camera

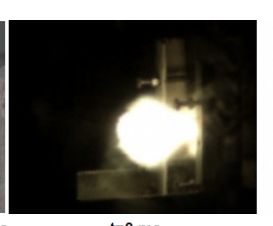

$\mathrm{t}=0 \mathrm{~ms}$ $\mathrm{t}=0.33 \mathrm{~ms}$

(c) $v=1249 \mathrm{~m} / \mathrm{s}$

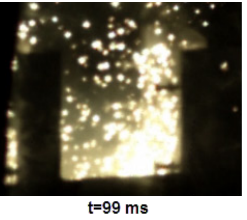

$\mathrm{t}=0.66 \mathrm{~ms}$ $\mathrm{t}=99 \mathrm{~ms}$

Figure 4: The processes of reactive fragments impacting the target plate 


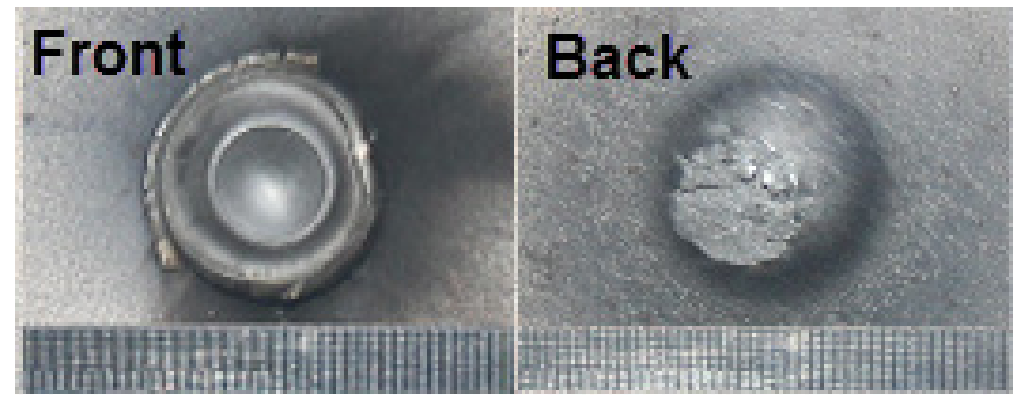

(a) Reactive fragments $v=593 \mathrm{~m} / \mathrm{s}$

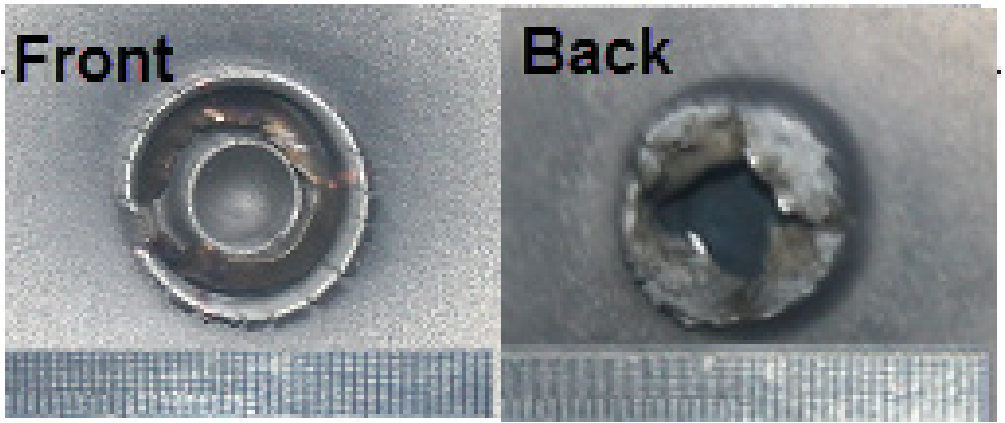

(b) Reactive fragments $v=731 \mathrm{~m} / \mathrm{s}$

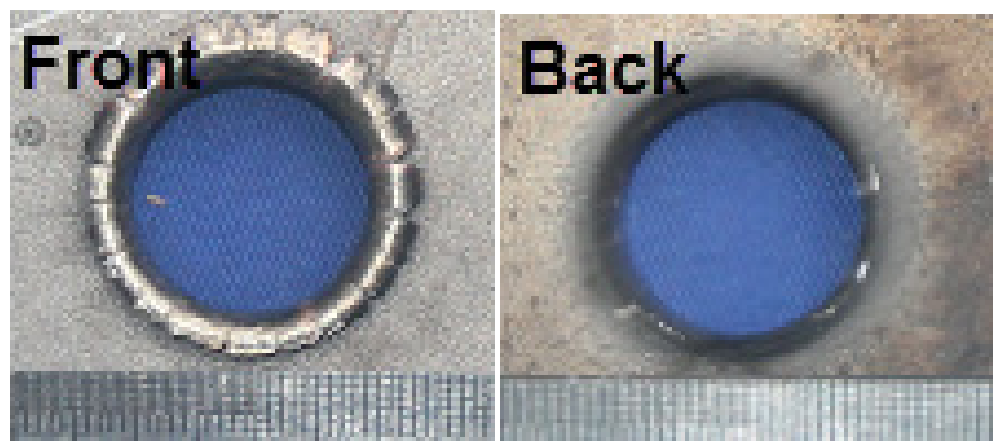

(c) Reactive fragments $v=1249 \mathrm{~m} / \mathrm{s}$

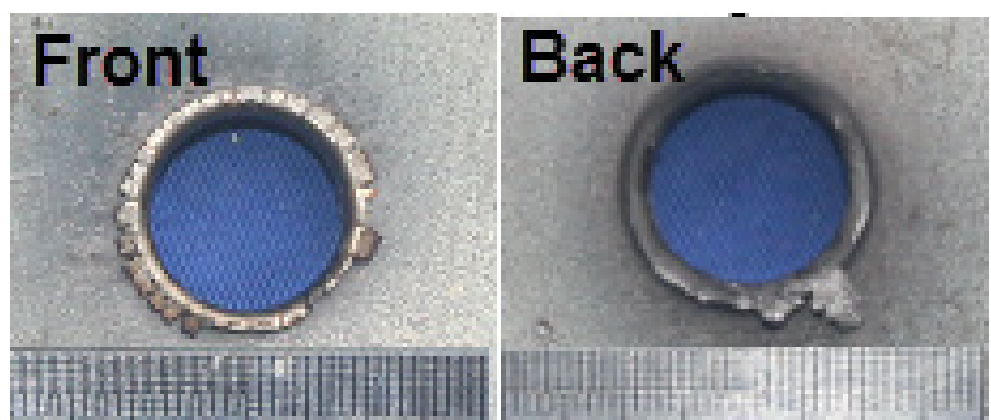

(d) Steel fragments $v=854 \mathrm{~m} / \mathrm{s}$

Figure 5: The typical images of the penetrated holes into the steel targets, effects of reactive and steel fragments on the targets 
The change curve of the targets aperture with the impact velocity of the fragments, which had been obtained by Origin software based on the linear fitting of least-squares method, was shown in Figure 6. It could be known that diameter of penetrated holes had been increased with the increase of the impact velocity and the gradient of the linear fitting line of the reactive fragments was basically the same as that of the steel fragments. The diameter of holes, penetrated with the reactive fragments had been increases by $10 \%$ when the targets was impacted by the two types fragments at the same velocity.

Table 1: The Perforated Hole Diameter of Steel plate

\begin{tabular}{lllll}
\hline Number & Fragment & $\mathbf{v} /(\mathbf{m} / \mathbf{s})$ & $\mathbf{d} /(\mathbf{m m})$ & $\boldsymbol{N}=\boldsymbol{d} / \boldsymbol{d}_{\mathbf{0}}$ \\
\hline 1 & 500 & - & - \\
2 & 651 & 14.5 & 1.21 \\
3 & 710 & 14.9 & 1.24 \\
4 & \multirow{2}{*}{ Steel Fragment } & 854 & 15.7 & 1.31 \\
5 & 1020 & 16.9 & 1.41 \\
6 & 1193 & 16.8 & 1.40 \\
7 & 1300 & 16.9 & 1.41 \\
\hline 8 & 593 & - & - \\
9 & 610 & - & - \\
10 & 619 & 16.5 & 1.38 \\
11 & 731 & 16.7 & 1.39 \\
12 & 732 & 16.5 & 1.38 \\
13 & 830 & 17.8 & 1.48 \\
14 & 864 & 17.9 & 1.49 \\
15 & & 16.8 & 1.40 \\
16 & Reactive Fragment & 17.2 & 1.43 \\
17 & 871 & 17.6 & 1.47 \\
18 & 1030 & 17.8 & 1.48 \\
19 & & 18.6 & 1.55 \\
20 & 1032 & 19.8 & 1.65 \\
21 & & 19.3 & 1.61 \\
\hline
\end{tabular}

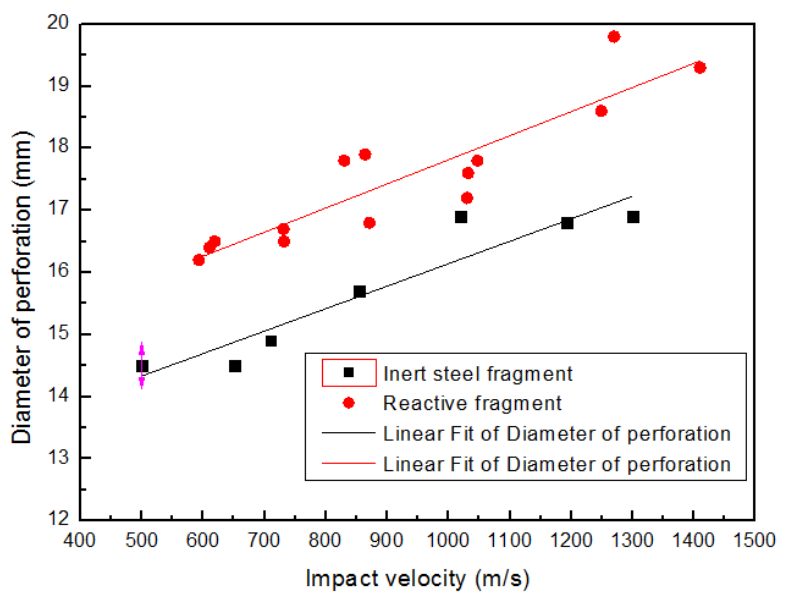

Figure 6: The relationship between diameter of the penetrated holes and impact velocity 


\subsubsection{Damage effects on the aluminum targets}

The typical images of the holes, penetrated with the two types of fragments to the aluminum targets was shown in Figure 7. The after-effect damages could not been resulted under the condition that the impact velocity of the fragments (v) was $593 \mathrm{~m} / \mathrm{s}$ and $731 \mathrm{~m} / \mathrm{s}$. When the impact velocity was $1249 \mathrm{~m} / \mathrm{s}$, the remarkable reaming with the irregular shapes could be produced by reactive fragments impacting the after-effect aluminum targets, accompanied by the strong plastic flanging effects. The diameter circular holes, penetrated with the steel fragments impacting the after-effect targets, was slightly larger than the diameter of the steel fragments.

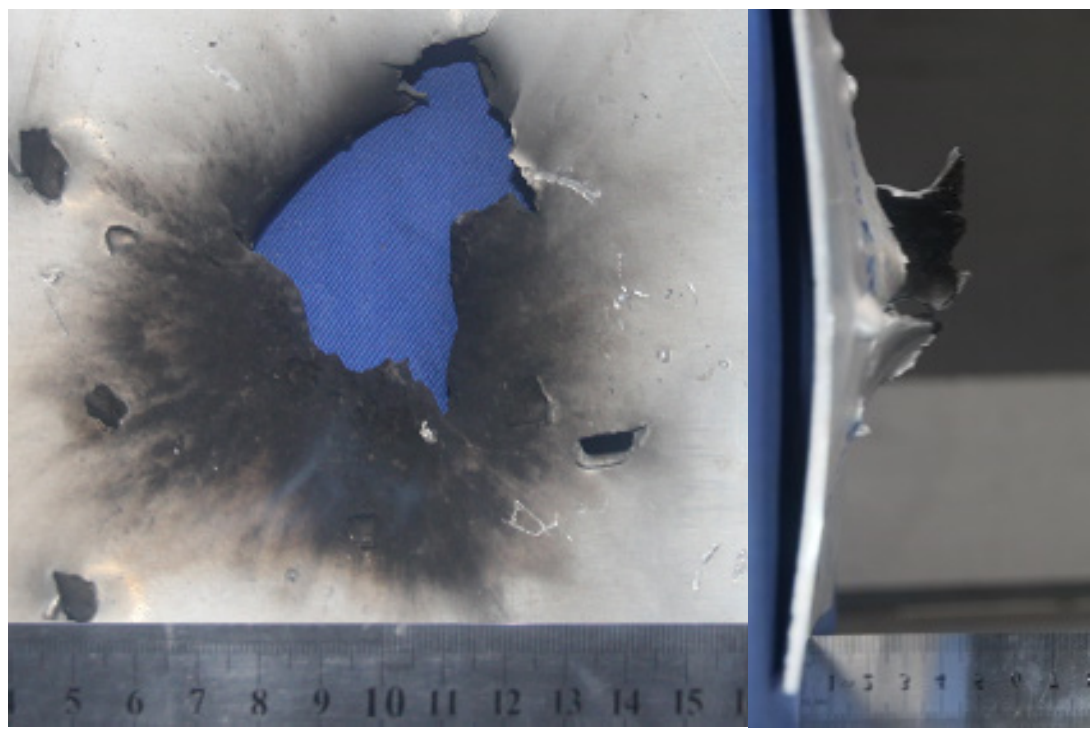

(a) Reactive fragment $\mathrm{v}=1249 \mathrm{~m} / \mathrm{s}$

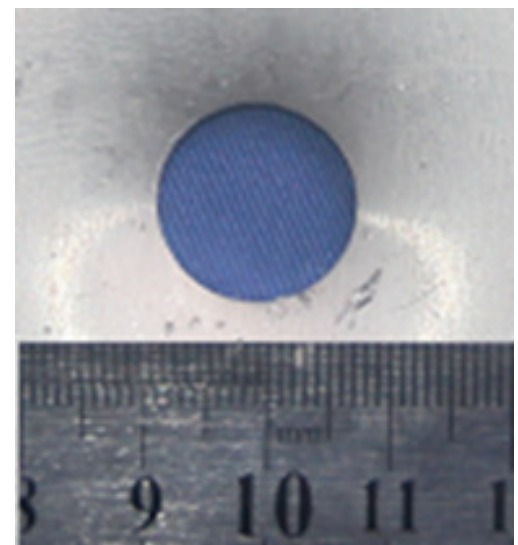

(b) Steel fragment $v=854 \mathrm{~m} / \mathrm{s}$

Figure 7: The typical images of the penetrated holes to the aluminum targets 
The holes with the irregular shapes had been penetrated to the aluminum targets under the condition that the impact velocity of the fragments was greater than a certain threshold. Therefore, the area of the penetrated holes to the aluminum targets could been used as the parameter to characterize the after-effect power of the fragments. The area of the holes, penetrated the after-effect aluminum targets by this experiment, was shown in Table 2.

Table 2: The Perforation Hole Area of the aluminum targets

\begin{tabular}{lllll}
\hline Number & Fragment & $\mathbf{v} /(\mathbf{m} / \mathbf{s})$ & $\boldsymbol{S} /(\mathbf{m m})$ & $\boldsymbol{\eta}=\boldsymbol{S} / \boldsymbol{S}_{\mathbf{0}}$ \\
\hline 1 & 500 & - & - \\
2 & 651 & 201 & 1.78 \\
3 & 710 & 209 & 1.85 \\
4 & Steel Fragment & 854 & 214 & 1.89 \\
5 & & 1020 & 227 & 2.01 \\
6 & 1193 & 227 & 2.01 \\
7 & 1300 & 227 & 2.01 \\
\hline 8 & 593 & - & - \\
9 & 610 & - & - \\
10 & 619 & - & - \\
11 & 731 & - & - \\
12 & 732 & - & - \\
13 & & 269 & 2.38 \\
14 & & 41 & 0.36 \\
15 & Reactive Fragment & 864 & 149 & 1.32 \\
16 & 871 & 615 & 1.81 \\
17 & 1030 & 354 & 3.13 \\
18 & & 1032 & 367 & 3.25 \\
19 & & 1047 & 956 & 12.42 \\
20 & & 1351 & 8.46 \\
21 & & 1249 & & 11.96 \\
\hline
\end{tabular}

$S$ represented the area of the penetrated holes to the after-effect aluminum targets, $S_{0}$ represented the initial section area of the fragments. In order to characterize the damageenhanced effects on the after-effect aluminum targets, command that.

$$
\eta=S / S_{0}
$$

$\eta$ represented the multiples of the penetrated holes area to the initial section area of the fragments. It could be known that the damage enhanced effects could reach 12.42 times to the initial section area of the reactive fragments and that of steel fragments was twice only.

The change curve of the area of the penetrated holes to the after-effect aluminum targets with the impact velocity of the fragments was shown in Figure 8. The area of the holes, penetrated to the after-effect aluminum targets with the inert steel fragments, had been remained about the same with the increase of impact velocity. However, the area of the holes, penetrated with the reactive fragments, had been improved significantly with the increase of impact velocity when the impact velocity was greater than a certain threshold. The intersection of the two curves in Figure 8 has been taken as the velocity threshold of the damage enhanced effects and the impact velocity $\left(v_{c r}\right)$ was $900 \mathrm{~m} / \mathrm{s}$. 


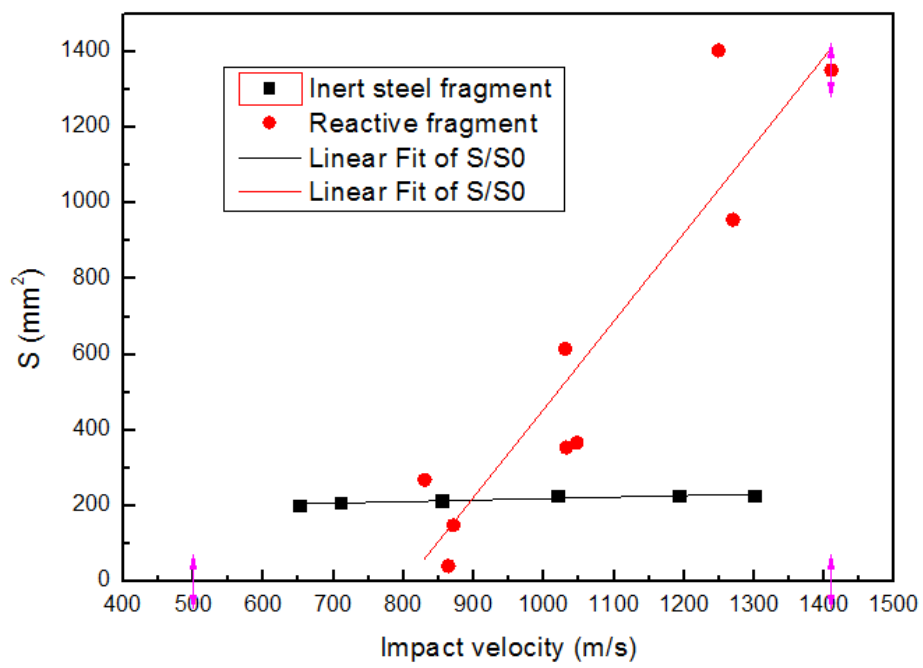

Figure 8: The relationship between hole area and impact velocity of the aluminum targets

\subsection{The mechanism of the damage enhanced effects for the reactive fragments}

\subsubsection{The ballistic-limited velocity of the reactive fragments}

The ballistic-limited velocity, denoted $v_{50}$, is an important index to evaluate the penetrating power of the fragments that refers to the average value of the maximum velocity of fragments penetrating the targets and the minimum velocity of fragments penetrating the targets completely. $v_{50}$ had been proved to obey normal distribution with a large number of experiments. When the number of unpenetrated fragments is greater or less than the penetrated number, the calculation formula of $v_{50}$ is as follows [10]:

$$
\begin{aligned}
& v_{50}=v_{\mathrm{A}}+\frac{N_{\mathrm{P}}-N_{\mathrm{c}}}{N_{\mathrm{P}}+N_{\mathrm{c}}}\left(v_{\mathrm{pmax}}-v_{\mathrm{A}}\right) \\
& v_{50}=v_{\mathrm{A}}+\frac{N_{\mathrm{c}}-N_{\mathrm{p}}}{N_{\mathrm{P}}+N_{\mathrm{c}}}\left(v_{\mathrm{A}}-v_{\mathrm{pmin}}\right)
\end{aligned}
$$

In the formula, $v_{A}$ represented the average value of all velocities in the mixed zone that the targets had been penetrated and unpenetrated. $N_{P}$ represented the number of the unpenetrated fragments and $N_{c}$ represented the penetrated fragments in the mixed zone. $v_{\text {pmax }}$ represented the maximum velocity of the unpenetrated fragments. $v_{p m i n}$ represented the minimum velocity of the penetrated fragments. Based on the experimental data in this study, it could be concluded that the ballistic-limited velocity $\left(v_{50}\right)$ of the coated reactive fragments was $709 \mathrm{~m} / \mathrm{s}$.

The empirical formula could be used to calculate the ballistic-limited velocity $\left(v_{50}\right)$ of the inert steel fragments [11],

$$
v_{50}=k \cdot(\mathrm{T} \overline{\mathrm{A}})^{\alpha} \cdot m_{f}^{\beta}
$$


In the formula, $T$ represented the thickness of the targets plates. $\bar{A}$ represented the average area of the fragments impacting the targets. $m_{f}$ represented the mass of the fragments. $k, \alpha$ and $\beta$ were the empirical coefficients related to the materials of the targeted plates. In this study, the empirical coefficients had been set as that $k$ was $6942, \alpha$ was 0.906 and $\beta$ was0.906 . It could be concluded that the ballistic-limited velocity $\left(v_{50}\right)$ of the insert steel fragments was $532 \mathrm{~m} / \mathrm{s}$.

It follows that the ballistic-limited velocity of the reactive fragments was 1.3 times than that of the insert steel fragments with the same mass and size that means the penetration ability of active fragments had been weakened by $33 \%$. The probable reasons was that the upsetting deformation had been enlarged and the penetration resistance had been increased due to the strength of the reactive fragments is lower than that of steel. As a result, the steel targeted plates with the same thickness could be penetrated at a higher impact velocity.

3.3.2. The mechanism of the damage enhanced effects for the reactive fragments The damage enhanced effects were resulted constitutionally from the penetration coupled damage of the fragments. The mechanism of the damage enhanced effects for the coated reactive fragments had been analyzed further in this study.

The residual velocity of the fragments impacting the targets could be calculated with the formula as follows [11]:

$$
v_{r}=v-v_{50}
$$

The velocity threshold and $v_{50}$ ballistic-limited velocity of the damage enhanced effects could be calculated building on the above studies. Substituting into the formula (6), it could be concluded that the residual velocity of the fragments was $181 \mathrm{~m} / \mathrm{s}$. Based on the analysis of the stress wave and threshold velocity of the reactive materials impacting the targets in the literature [12], the velocity was equivalent to the threshold velocity of the "exposed" reactive fragments impacting the targets and the perforation capabilities of fragments would be improved significantly with increased velocity further. However, the experimental results of the above literature indicated that the damage effects of "perforation superimposing a strong plastic deformation" that had been produced by these experiments was insufficient to be achieved only by relying on this part of energy. Combined with observation results by the high-speed camera in this paper, it was found that some inner core reactive materials had been initiated reactions to release energy after the steel targets had been penetrated by the coated active fragments. Thus, it could be seen that the energy, triggering the damage enhanced effects of the coated reactive fragments, was composed of two parts. Part of the energy had been released from the reactions which had been initiated by the shear fracture of the reactive materials in the internal stress action, resulted from the reactive fragments penetrating the steel targets. The other part of the energy had been released from the reactions which had been initiated by the comprehensive actions of penetration and shear, resulted from the unbroken reactive materials continue impacting the after-effect targets. With the coupling of two parts of damage energy, the damage enhanced effects of "perforation superimposing a strong plastic deformation" on the after- effect targets had been produced. Since the velocity of energy released and the completeness of the reactions for the reactive materials was closely related to the impact velocity, the strength of the damage enhanced effects was enhanced with the increase of the impact velocity when it was greater than or equal to $900 \mathrm{~m} / \mathrm{s}$. 


\section{CONCLUSION}

For research the penetration capability and the after-damage-enhanced effects of the steel fragments and coated reactive fragments, the impact test of the two types of fragments with the same mass and size penetrating the steel targets had been completed adopting ballistic rifle. The main conclusions were as follows:

1) The integral strength of the coated reactive fragments was lower than that of the inert steel fragments, which would lead to the reduce the penetration capability. The v50 ballisticlimited velocity of the coated reactive fragments impacting the $6 \mathrm{~mm}$ Q235A steel target, was $709 \mathrm{~m} / \mathrm{s}$ and it was 1.33 times than that of the steel fragments with the same mass and size. The perforation diameter of the steel targets was greater by the coated reactive fragments than the insert steel fragments perforated. The perforation diameter, resulted the reactive fragments, had been increases by $10 \%$ when the targets was impacted by the two types fragments at the same velocity.

2) The expansion enhanced effect on the after effect-targets could be produced in the condition that the impact velocity of the coated reactive fragments should be greater than $900 \mathrm{~m} / \mathrm{s}$ while the residual velocity should be greater than $181 \mathrm{~m} / \mathrm{s}$, and that the expansion enhanced effects had been strengthened along with the impact velocity increased. The probable reasons of the damage enhanced effects had been analyzed that the coupled damage effects had been resulted from two parts energy. Part of the energy had been released from the reactions which had been initiated by the shear fracture of the reactive materials in the internal stress action, resulted from the reactive fragments penetrating the steel targets. The other part of the energy had been released from the reactions which had been initiated by the comprehensive actions of penetration and shear, resulted from the unbroken reactive materials continue impacting the after-effect targets.

\section{REFERENCES}

[1] Li Shuang, Wang Lixia. Study on reactive material warhead propped by MDBA company[J]. Aero Weaponry, 2016(2):76-76.

[2] Peng jun, Yuan Baohui, Chen Jin, etc. The Damage enhancement reactive projectile technology[J]. Aerodynamic missile journal, 2017(8):84-88.

[3] Ronald A. Advanced energetic materials[R], Washington DC: National Academies Press, 2004.

[4] YANG Yi, ZHENG Ying, WANG Kun. Development progress of high density reactive materials and their damage effect [J]. Ordnance Material Science And Engineering, 2013,36(4):81-85.

[5] SHUAI Jun-feng, JIANG Jian-wei, WANG Shu-you, etc. Compound Reactive Fragment Penetrating Steel Target[J]. Chinese Journal of Energetic Materials, 2009,17(6):722-725.

[6] LU Zhonghua, SUN Wenxu, LUO Zhiheng, etc. Penetration Effect of Reactive Fragment on Medium Thickness Aluminum Alloy Target Aimed at[J]. Science and Technology Review,2013,31(17):46-50.

[7] CHEN Jin, CHEN Yuan-jian, YUAN Bao-hui, etc. Experimental Investigation on Penetration Behavior of Reactive Fragment against Steel Plates[J]. Science Technology and Engineering, 2014,14(35):52-55.

[8] Fengyue Xu, Baoqun Geng, Xuepeng Zhang, etc. Experimental Study on behind-Plate Overpressure Effect by Reactive Material Projectile[J]. Propellants, Explosives, Pyrotechnics, 2017, (42): 192-197. 
[9] Brett Sorensen. High-Velocity impact of Al/PTFE Projectiles on structural Aluminum Armor [J]. Procedia Engineering, 2015, (103): 569-576.

[10] Mao Liang, Wang Hua, Jiang Chun-lan, etc. Experimental Research on Tungsten Alloy Spherical Fragment Penetrating DFRP Target Plates[J].Transactions of Beijing Institute of Technology, 2016,36 (4):348-353.

[11]WANG Shu-shan. Terminal Effects [M]. The second edition. Beijing: Science Press, 2019.

[12]XIAO Yan-wen, XU Feng-yue, YU Qing-bo, etc. Experimental Research on Behavior of Active Material Projectile with Steel-like Density Impacting Aluminum Target[J]. Acta Armamentarii, 2016,37 (06):1016-1022. 